\title{
ON THE FEASIBILITY OF DETERMINING LIGHT-SOURCE DIRECTION FROM AN IMAGE
}

\author{
BY \\ WOJCIECH CHOJNACKI (Department of Computer Science, University of Adelaide, Australia), \\ MICHAEL J. BROOKS (Department of Computer Science, University of Adelaide, Australia), \\ AND *
}

DANIEL GIBBINS (Centre for Sensor Signal and Information Processing, Technology Park, The Levels, Australia)

\begin{abstract}
Various computational techniques have been developed that perform reasonably well in inferring shape from shading. However, these techniques typically require substantial prerequisite information if they are to evolve an estimate of surface shape. It is therefore interesting to consider how depth might be inferred from shading information without prior knowledge of various scene conditions. One approach has been to undertake a pre-processing step of estimating the light-source direction, thereby providing input to the computation of shape from shading. In this paper, we present evidence that a versatile light-source-direction estimator is unattainable.
\end{abstract}

1. Introduction. The shape-from-shading problem may under certain assumptions be expressed mathematically as a first-order partial differential equation [4]. Much information is needed to be able to formulate such an image irradiance equation, a principal requirement being an appropriate reflectance map [6] relating surface orientation to image brightness. Without this information, the problem cannot be posed, let alone solved. Even if the image irradiance equation can be formulated, it may be difficult to solve, may have many solutions, or may even have no solutions [1]. A major challenge in computer vision is to develop a versatile shape-from-shading algorithm able to operate effectively in the absence of substantial prerequisite information [5]. Such an algorithm has so far proven elusive.

Most shape-from-shading schemes need to be given prior information that describes light-source configuration and surface reflectance properties. Various strategies have

Received June 13, 1995.

1991 Mathematics Subject Classification. Primary 68T10; Secondary 35F20.

Key words and phrases. Shape from shading, image irradiance equation, light-source direction, feasibility, estimation.

E-mail address: wojtek@cs.adelaide.edu.au

E-mail address: mjbøcs.adelaide.edu.au

E-mail address: danny@cssip.edu.au 
been employed in an attempt to reduce the prerequisite requirements of shading algorithms. One approach, first adopted by Pentland [8], has been to estimate the direction of the "sun" (light source) prior to determining shape. In this paper, we argue that this approach is of limited applicability, and present evidence that a truly versatile lightsource-direction estimator is unattainable if undertaken as a pre-process to shape from shading. To our knowledge, this is the first meta-analysis undertaken in this area.

Note that we discuss the source-direction estimation problem from the local point of view, restricting our attention to the information content of interior patches of image shading. Global aspects of the estimation problem, such as the potential value of occluding boundary information, are not considered.

2. Estimating source direction. Estimation of lighting conditions from a given image requires some understanding of how the depicted surface scatters light. For reasons of tractability, we shall adopt the Lambertian model with unit albedo that describes lightscattering properties of perfectly matte surfaces. Assume that a surface represented by the graph of a $C^{2}$ function $f$ is viewed from the direction specified by the vector $\boldsymbol{e}=(0,0,1)$. Suppose that the surface is illuminated by an infinitely distant sun from the direction determined by the unit vector $l=\left(l_{1}, l_{2}, l_{3}\right)$. Then the image of the surface over the corresponding image domain in the $(x, y)$-plane has irradiance $E$ that takes on values of the interval $(0,1]$ and satisfies the image irradiance equation

$$
R_{l}\left(\frac{\partial f}{\partial x}, \frac{\partial f}{\partial y}\right)=E(x, y)
$$

where $R_{l}$ is the Lambertian reflectance map

$$
R_{l}(p, q)=\frac{-l_{1} p-l_{2} q+l_{3}}{\sqrt{p^{2}+q^{2}+1}} .
$$

The light-source-direction estimation problem may now be expressed simply as the need to determine $l$ given $R_{l}$ and $E$, and not given $f$.

In the following, we confine our attention to local aspects of the light-source-direction estimation problem, assessing whether an illuminant vector can be derived from shading a neighbourhood (of unspecified form and size) of a point in an image. As is common practice in studying local properties of functions, we shall employ the language of germs (cf. [9, Chapter 1, Paragraph 4]). Informally speaking, two functions define, at a given point, the same germ if they are equal on some open neighbourhood of that point. Recourse to the notion of a germ enables extraction of the essential characteristics of a function in a vicinity of a point. We start by presenting elements of the terminology of germs.

Let $z$ be a point in the $(x, y)$-plane, and let $k$ be a nonnegative integer. Consider the set $\mathcal{P}_{z}^{k}$ consisting of pairs $(U, f)$, where $U$ is an open neighbourhood of $z$ and $f$ is a real function that possesses continuous partial derivatives of order $\leq k$ (when $k=0$, we assume $f$ to be continuous). Introduce a binary relation $\sim$ on this set by setting

$$
(U, f) \sim(V, g)
$$


if there exists an open neighbourhood $W$ of $z$ contained in $U \cap V$ such that $f$ coincides with $g$ on $W$. It is easily seen that $\sim$ is an equivalence relation; that is, the following conditions are satisfied:

(i) $(U, f) \sim(U, f)$;

(ii) $(U, f) \sim(V, g) \quad \Rightarrow \quad(V, g) \sim(U, f)$;

(iii) $(U, f) \sim(V, g) \wedge(V, g) \sim(W, h) \Rightarrow(U, f) \sim(W, h)$.

Given $(U, f) \in \mathcal{P}_{z}^{k}$, let $[f]_{z}$ be the set of all $(V, g) \in \mathcal{P}_{z}^{k}$ that are equivalent to $(U, f)$. $[f]_{z}$ is called the equivalence class of $(U, f)$ for $\sim$, or the germ of $f$ at $z$. All elements of a given equivalence class are equivalent to one another, as follows at once from the three properties of an equivalence relation. Furthermore, two equivalence classes either coincide or have no element in common. Thus $\sim$ determines a decomposition of $\mathcal{P}_{z}^{k}$ into disjoint equivalence classes. Each element of the class is called a representative of the class. Designate by $\mathcal{C}_{z}^{k}$ the set of all equivalence classes for $\sim$. For $k=0$, contract the notation $\mathcal{C}_{z}^{k}$ to $\mathcal{C}_{z}$. Given $[f]_{z} \in \mathcal{C}_{z}^{k}$, define the value $[f]_{z}(z)$ of $[f]_{z}$ at $z$ as follows: Starting from a representative $(U, f)$ of $[f]_{z}$, set $[f]_{z}(z)=f(z)$; by the definition of an equivalence relation, this is independent of any particular choice of $(U, f)$ within the equivalence class $[f]_{z}$. A germ $[f]_{z} \in \mathcal{C}_{z}^{k}$ is positive if $[f]_{z}(z)>0$. Any two germs in $\mathcal{C}_{z}^{k}$ can be added and multiplied, and also germs in $\mathcal{C}_{z}^{k}$ can be multiplied by scalars, the results remaining germs in $\mathcal{C}_{z}^{k}$. Indeed, given $[f]_{z},[g]_{z} \in \mathcal{C}_{z}^{k}$ and a real number $\lambda$, if $(U, f)$ and $(V, g)$ are representatives of $[f]_{z}$ and $[g]_{z}$, respectively, we can define $[f]_{z}+[g]_{z},[f]_{z}[g]_{z}$, and $\lambda[f]_{z}$ as the germs at $z$ with the respective representatives

$$
(U \cap V, f+g), \quad(U \cap V, f g), \quad(U, \lambda f) .
$$

This definition is clearly independent of the initial representatives chosen. If $[f]_{z}(z) \neq 0$, then $[f]_{z}$ can be inverted. In fact, if we let $(U, f)$ be a representative of $[f]_{z}$, then, by definition, $[f]_{z}(z)=f(z)$, and so there is an open neighbourhood $V \subset U$ of $z$ such that $f(w) \neq 0$ for each $w \in V$. Now the equivalence class of $(V, 1 / f)$ is the inverse of $[f]_{z}$. Germs in $\mathcal{C}_{z}^{k}$ with $k \geq 1$ can be differentiated, the derivatives of order $l(1 \leq l \leq k)$ being members of $\mathcal{C}_{z}^{k-l}$. Indeed, if $[f]_{z} \in \mathcal{C}_{z}^{k}$ with $k \geq 1$ has a representative $(U, f)$, we can naturally define $\partial[f]_{z} / \partial x$ and $\partial[f]_{z} / \partial y$ as the germs in $\mathcal{C}_{z}^{k-1}$ with the representatives $(U, \partial f / \partial x)$ and $(U, \partial f / \partial y)$. Once again this definition is independent of the initial representative chosen. Higher-order derivatives of $[f]_{z}$ can be defined analogously.

With these preliminaries, we can now introduce a number of definitions that will be of direct relevance in our analysis of the light-source-direction estimation problems.

Given a unit vector $l$ and a germ $[f]_{z} \in \mathcal{C}_{z}^{2}$, the image of $[f]_{z}$ from the direction $l$ is the germ $[E]_{z} \in \mathcal{C}_{z}^{1}$ defined by

$$
[E]_{z}=R_{l}\left(\frac{\partial[f]_{z}}{\partial x}, \frac{\partial[f]_{z}}{\partial y}\right)
$$

and the condition that $[E]_{z}$ be positive. (Note that already this definition makes use of all the algebraic and differential properties of germs described above.) A shape class at $z$ is any subset of $\mathcal{C}_{z}^{2}$. Given a shape class $\mathcal{S}_{z} \subset \mathcal{C}_{z}^{2}$, the corresponding image class $\mathcal{E}_{\mathcal{S}_{z}}$ is the family of all images of the elements of $\mathcal{S}_{z}$, that is,

$$
\mathcal{E}_{\mathcal{S}_{z}}=\left\{R_{l}\left(\frac{\partial[f]_{z}}{\partial x}, \frac{\partial[f]_{z}}{\partial y}\right): l \text { is a unit vector, }[f]_{z} \in \mathcal{S}_{z}, R_{l}\left(\frac{\partial[f]_{z}}{\partial x}, \frac{\partial[f]_{z}}{\partial y}\right)>0\right\} .
$$


A shape class $\mathcal{S}_{z} \subset \mathcal{C}_{z}^{2}$ is termed a uniqueness class at $z$ if, for any $[f]_{z},[g]_{z} \in \mathcal{S}_{z}$ and any unit vectors $l, l^{\prime}$,

$$
R_{l}\left(\frac{\partial[f]_{z}}{\partial x}, \frac{\partial[f]_{z}}{\partial y}\right)=R_{l^{\prime}}\left(\frac{\partial[g]_{z}}{\partial x}, \frac{\partial[g]_{z}}{\partial y}\right)>0 \quad \Rightarrow \quad l=l^{\prime}
$$

Observe that there is a one-to-one correspondence between elements of a given uniqueness class and the respective images in the image class associated with this uniqueness class. Thus, starting from a uniqueness class $\mathcal{S}_{z}$, we can define a light-source-direction estimator based on $\mathcal{S}_{z}$ as the function $\Lambda_{\mathcal{S}_{z}}$ which, to each member $[E]_{z}$ in the corresponding image class $\mathcal{E}_{\mathcal{S}_{z}}$, assigns a unique unit vector $\boldsymbol{l}$ for which (2) holds.

Light-source-direction estimators based on uniqueness classes can be used as follows. Given an image $E$ over a domain $\Omega$, suppose that, for some $z \in \Omega$, the germ of $E$ at $z$ is the image of a member of a given uniqueness class $\mathcal{S}_{z}$. This assumption effectively means that we have certain prior information about the nature of the shape that has generated the image in the vicinity of $z$. More precisely, we assume that, for some open neighbourhood $U \subset \Omega$ of $z$, the restriction of $E$ to $U$ has been generated by a shape $f$ such that the equivalence class of $(U, f)$ belongs to the uniqueness class $\mathcal{S}_{z}$ that is known beforehand. By applying the corresponding estimator $\Lambda_{\mathcal{S}_{z}}$ to $[E]_{z}$, we can determine the illuminant vector $\boldsymbol{l}$. Following this, we can proceed to solving (1) on the whole of $\Omega$. It is obvious that the success of such an approach relies strongly upon early recognition of various, possibly vast, uniqueness classes to which a germ of a sought-after shape might belong. The question of determining uniqueness classes is therefore considered next.

3. Uniqueness classes. We begin by exhibiting simple uniqueness classes, each of which consists of a single germ.

For a real $C^{2}$ function $f$ on an open subset $U$ of the $(x, y)$-plane, denote by $D(f)$ the continuous function on $U$ given by

$$
D(f)=\left|\begin{array}{cc}
\frac{\partial^{2} f}{\partial x^{2}} & \frac{\partial^{2} f}{\partial x \partial y} \\
\frac{\partial^{2} f}{\partial x \partial y} & \frac{\partial^{2} f}{\partial y^{2}}
\end{array}\right|
$$

Similarly, if $z$ is a point in the $(x, y)$-plane and $[f]_{z} \in \mathcal{C}_{z}^{2}$, denote by $D\left([f]_{z}\right)$ the germ in $\mathcal{C}_{z}$ defined as

$$
D\left([f]_{z}\right)=\left|\begin{array}{ll}
\frac{\partial^{2}[f]_{z}}{\partial x^{2}} & \frac{\partial^{2}[f]_{z}}{\partial x \partial y} \\
\frac{\partial^{2}[f]_{z}}{\partial x \partial y} & \frac{\partial^{2}[f]_{z}}{\partial y^{2}}
\end{array}\right|
$$

THEOREM 1 . Let $z$ be a point in the $(x, y)$-plane, and let $[f]_{z} \in \mathcal{C}_{z}^{2}$ satisfy

$$
D\left([f]_{z}\right)(z) \neq 0
$$

Then $\left\{[f]_{z}\right\}$ is a uniqueness class.

Note that (4) is equivalent to the condition that

$$
D(f)(z) \neq 0
$$


for any representative $(U, f)$ on $[f]_{z}$. Inequality $(5)$ is in turn equivalent to the requirement that the Gaussian curvature of the graph of $f$ over $U$ at the point $(z, f(z))$ be nonzero.

Proof of Theorem 1. Let $z$ be a point in the $(x, y)$-plane. Suppose that $[f]_{z} \in \mathcal{C}_{z}^{2}$ satisfies (4) and that

$$
R_{l}\left(\frac{\partial[f]_{z}}{\partial x}, \frac{\partial[f]_{z}}{\partial y}\right)=R_{l^{\prime}}\left(\frac{\partial[f]_{z}}{\partial x}, \frac{\partial[f]_{z}}{\partial y}\right)
$$

for some unit vectors $l, l^{\prime}$. Clearly, we can choose a representative $(U, f)$ of $[f]_{z}$ such that

$$
R_{l}\left(\frac{\partial f}{\partial x}, \frac{\partial f}{\partial y}\right)(w)=R_{l^{\prime}}\left(\frac{\partial f}{\partial x}, \frac{\partial f}{\partial y}\right)(w)
$$

for each $w \in U$. Then, for each $w \in U$,

$$
a \frac{\partial f}{\partial x}(w)+b \frac{\partial f}{\partial y}(w)=c
$$

where $a=l_{1}-l_{1}^{\prime}, b=l_{2}-l_{2}^{\prime}$, and $c=l_{3}-l_{3}^{\prime}$. Differentiating both sides of $(6)$ at $z$ with respect to $x$ and $y$, we obtain

$$
\begin{aligned}
& a \frac{\partial^{2} f}{\partial x^{2}}(z)+b \frac{\partial^{2} f}{\partial x \partial y}(z)=0 \\
& a \frac{\partial^{2} f}{\partial x \partial y}(z)+b \frac{\partial^{2} f}{\partial y^{2}}(z)=0
\end{aligned}
$$

Taking into account that (4) implies (5), we infer from (7) that $a=b=0$. Hence $l=l^{\prime}$, which establishes implication (3).

Our next result will provide us with a large supply of one-element uniqueness classes. While relevant in the further development, these uniqueness classes will by no means exhaust the family of all one-element uniqueness classes.

THEOREM 2. Let $z$ be a point in the $(x, y)$-plane, let $F$ be a holomorphic function on an open neighbourhood of $z$, and let $u$ and $v$ be the real and imaginary parts of $F$, respectively. For each $\alpha \in[0,2 \pi)$, let

$$
g_{\alpha}=u \cos \alpha+v \sin \alpha .
$$

Then, for each $\alpha \in[0,2 \pi)$,

$$
D\left(g_{\alpha}\right)=-\left|F^{\prime \prime}\right|^{2} .
$$

In particular, if $F^{\prime \prime}(z) \neq 0$, then each $\left\{\left[g_{\alpha}\right]_{z}\right\}$ is a uniqueness class.

Proof. Since $F$ is holomorphic, $u$ and $v$ satisfy the Cauchy-Riemann equations:

$$
\begin{aligned}
& \frac{\partial u}{\partial x}=\frac{\partial v}{\partial y} \\
& \frac{\partial u}{\partial y}=-\frac{\partial v}{\partial x}
\end{aligned}
$$


It follows that

$$
\begin{aligned}
& \frac{\partial^{2} u}{\partial x^{2}}=-\frac{\partial^{2} u}{\partial y^{2}}=\frac{\partial^{2} v}{\partial x \partial y} \\
& \frac{\partial^{2} v}{\partial x^{2}}=-\frac{\partial^{2} v}{\partial y^{2}}=-\frac{\partial^{2} u}{\partial x \partial y}
\end{aligned}
$$

Hence

$$
F^{\prime \prime}=\frac{\partial^{2} u}{\partial x^{2}}+i \frac{\partial^{2} v}{\partial x^{2}}=\frac{\partial^{2} u}{\partial x^{2}}-i \frac{\partial^{2} u}{\partial x \partial y}
$$

and further

$$
\left|F^{\prime \prime}\right|^{2}=\left(\frac{\partial^{2} u}{\partial x^{2}}\right)^{2}+\left(\frac{\partial^{2} u}{\partial x \partial y}\right)^{2}=-\frac{\partial^{2} u}{\partial x^{2}} \cdot \frac{\partial^{2} u}{\partial y^{2}}+\left(\frac{\partial^{2} u}{\partial x \partial y}\right)^{2}=-D(u) .
$$

By (8) and (10), for each $\alpha \in[0,2 \pi)$,

$$
\begin{aligned}
& \frac{\partial g_{\alpha}}{\partial x}=\frac{\partial u}{\partial x} \cos \alpha-\frac{\partial u}{\partial y} \sin \alpha \\
& \frac{\partial g_{\alpha}}{\partial y}=\frac{\partial u}{\partial y} \cos \alpha+\frac{\partial u}{\partial x} \sin \alpha
\end{aligned}
$$

Hence

$$
\begin{aligned}
\frac{\partial^{2} g_{\alpha}}{\partial x^{2}} & =\frac{\partial^{2} u}{\partial x^{2}} \cos \alpha-\frac{\partial^{2} u}{\partial x \partial y} \sin \alpha \\
\frac{\partial^{2} g_{\alpha}}{\partial x \partial y} & =\frac{\partial^{2} u}{\partial x \partial y} \cos \alpha-\frac{\partial^{2} u}{\partial y^{2}} \sin \alpha=\frac{\partial^{2} u}{\partial x \partial y} \cos \alpha+\frac{\partial^{2} u}{\partial x^{2}} \sin \alpha \\
\frac{\partial^{2} g_{\alpha}}{\partial y^{2}} & =\frac{\partial^{2} u}{\partial y^{2}} \cos \alpha+\frac{\partial^{2} u}{\partial x \partial y} \sin \alpha
\end{aligned}
$$

Now

$$
\begin{aligned}
D\left(g_{\alpha}\right) & =\left|\begin{array}{ll}
\frac{\partial^{2} u}{\partial x^{2}} \cos \alpha-\frac{\partial^{2} u}{\partial x \partial y} \sin \alpha & \frac{\partial^{2} u}{\partial x \partial y} \cos \alpha+\frac{\partial^{2} u}{\partial x^{2}} \sin \alpha \\
\frac{\partial^{2} u}{\partial x \partial y} \cos \alpha-\frac{\partial^{2} u}{\partial y^{2}} \sin \alpha & \frac{\partial^{2} u}{\partial x \partial y} \sin \alpha+\frac{\partial^{2} u}{\partial y^{2}} \cos \alpha
\end{array}\right| \\
& =\left|\begin{array}{ll}
\frac{\partial^{2} u}{\partial x^{2}} & \frac{\partial^{2} u}{\partial x \partial y} \\
\frac{\partial^{2} u}{\partial x \partial y} & \frac{\partial^{2} u}{\partial y^{2}}
\end{array}\right| \cdot\left|\begin{array}{cc}
\cos \alpha & \sin \alpha \\
-\sin \alpha & \cos \alpha
\end{array}\right| \\
& =D(u),
\end{aligned}
$$

which together with (11) establishes (9).

As an example of a specific use of the theorem established, let $z=(a, b)$ and $F(w)=$ $(w-z)^{2} / 2$. With the terminology from Theorem 2 , we have

$$
u(x, y)=\frac{1}{2}\left((x-a)^{2}-(y-b)^{2}\right), \quad v(x, y)=(x-a)(y-b)
$$

and, for each $\alpha \in[0,2 \pi)$,

$$
g_{\alpha}(x, y)=\frac{1}{2}\left((x-a)^{2}-(y-b)^{2}\right) \cos \alpha+(x-a)(y-b) \sin \alpha .
$$

Since $F^{\prime \prime}=1$, it follows from Theorem 2 that, for each $\alpha \in[0,2 \pi),\left\{\left[g_{\alpha}\right]_{z}\right\}$ is a uniqueness class.

We now proceed to discuss the existence of uniqueness classes containing more than one element. A natural way of building such classes, at a given point, would be to combine 
one-element uniqueness classes into bigger families (for example, each such singleton set might be formed by a germ satisfying (4)). This procedure will work only for certain choices of germs. It turns out that the set-theoretic sum of two one-element uniqueness classes will in general fail to produce a uniqueness class. To illustrate this phenomenon, we shall need the following result:

TheOREM 3. Let $z$ be a point in the $(x, y)$-plane, let $F$ be a holomorphic function on an open neighbourhood of $z$, and let $u$ and $v$ be the real and imaginary parts of $F$, respectively. For each $\alpha \in[0,2 \pi)$, let $g_{\alpha}$ be given by (8). Then $\left\{\left[g_{\alpha}\right]_{z},\left[g_{\beta}\right]_{z}\right\}$ is not a uniqueness class whenever $\alpha, \beta \in[0,2 \pi)$ are such that $\alpha \neq \beta$.

Proof. Let $\boldsymbol{l}=\left(l_{1}, l_{2}, l_{3}\right)$ be a unit vector such that

$$
\begin{gathered}
0<l_{1}^{2}+l_{2}^{2}<1, \\
-l_{1} \frac{\partial u}{\partial x}(z)-l_{2} \frac{\partial u}{\partial y}(z) \geq 0,
\end{gathered}
$$

and $l_{3}>0$. Then

$$
-l_{1} \frac{\partial u}{\partial x}(z)-l_{2} \frac{\partial u}{\partial y}(z)+l_{3}>0 .
$$

For each $\alpha \in[0,2 \pi)$, let $l_{\alpha}=\left(l_{1}^{(\alpha)}, l_{2}^{(\alpha)}, l_{3}^{(\alpha)}\right)$ be the unit vector given by

$$
\begin{aligned}
& l_{1}^{(\alpha)}=l_{1} \cos \alpha-l_{2} \sin \alpha, \\
& l_{2}^{(\alpha)}=l_{1} \sin \alpha+l_{2} \cos \alpha, \\
& l_{3}^{(\alpha)}=l_{3} .
\end{aligned}
$$

By (12), for each $\alpha \in[0,2 \pi)$,

$$
-l_{1}^{(\alpha)} \frac{\partial g_{\alpha}}{\partial x}-l_{2}^{(\alpha)} \frac{\partial g_{\alpha}}{\partial y}+l_{3}^{(\alpha)}=-l_{1} \frac{\partial u}{\partial x}-l_{2} \frac{\partial u}{\partial y}+l_{3} .
$$

In view of (12),

$$
\left(\frac{\partial g_{\alpha}}{\partial x}\right)^{2}+\left(\frac{\partial g_{\alpha}}{\partial y}\right)^{2}=\left(\frac{\partial u}{\partial x}\right)^{2}+\left(\frac{\partial u}{\partial y}\right)^{2} .
$$

This together with (16) shows that

$$
R_{l_{\alpha}}\left(\frac{\partial g_{\alpha}}{\partial x}, \frac{\partial g_{\alpha}}{\partial y}\right)=R_{l}\left(\frac{\partial u}{\partial x}, \frac{\partial u}{\partial y}\right)
$$

and further, on account of (15),

$$
R_{l_{\alpha}}\left(\frac{\partial g_{\alpha}}{\partial x}, \frac{\partial g_{\alpha}}{\partial y}\right)(z)>0
$$

Hence

$$
R_{l_{\alpha}}\left(\frac{\partial\left[g_{\alpha}\right]_{z}}{\partial x}, \frac{\partial\left[g_{\alpha}\right]_{z}}{\partial y}\right)>0
$$

and furthermore, for any $\alpha, \beta \in[0,2 \pi)$,

$$
R_{l_{\alpha}}\left(\frac{\partial\left[g_{\alpha}\right]_{z}}{\partial x}, \frac{\partial\left[g_{\alpha}\right]_{z}}{\partial y}\right)=R_{l_{\beta}}\left(\frac{\partial\left[g_{\beta}\right]_{z}}{\partial x}, \frac{\partial\left[g_{\beta}\right]_{z}}{\partial y}\right) .
$$


We see that the image of $\left[g_{\alpha}\right]_{z}$ from the direction $\boldsymbol{l}_{\alpha}$ coincides with the image of $\left[g_{\beta}\right]_{z}$ from the direction $\boldsymbol{l}_{\beta}$. By (14), the 2-vector $\left(l_{1}, l_{2}\right)$ is nonzero. Thus if $\alpha, \beta \in[0,2 \pi)$ are such that $\alpha \neq \beta$, then $\boldsymbol{l}_{\alpha} \neq \boldsymbol{l}_{\beta}$, and so $\left[g_{\alpha}\right]_{z}$ and $\left[g_{\beta}\right]_{z}$ cannot belong to a common uniqueness class.

Now, given a point $z$ in the $(x, y)$-plane, fix a holomorphic function $F$ in an open neighbourhood of $z$ such that (5) holds (we can take $F(w)=(w-z)^{2} / 2$, for example). Using the terminology and result of Theorem 2 , we see that, for each $\alpha \in[0,2 \pi),\left[g_{\alpha}\right]_{z}$ is a uniqueness class. However, by Theorem 3, for any pair $\alpha, \beta \in[0,2 \pi)$ with $\alpha \neq \beta$ the set $\left\{\left[g_{\alpha}\right]_{z},\left[g_{\beta}\right]_{z}\right\}$ is not a uniqueness class. Thus the sum of two one-element uniqueness classes fails in general to be a uniqueness class.

The previous paragraph notwithstanding, there exist uniqueness classes different from singletons. Such classes can be built from elementary blocks provided that one of two natural compatibility conditions, stated below, is satisfied. To formulate these conditions, suppose that $\left\{\mathcal{S}_{z}^{i}\right\}_{i \in I}$ is an indexed family of pairwise distinct uniqueness classes at a given point $z$. Consider the following:

(A) For $[f]_{z} \in \mathcal{S}_{z}^{i}$ and $[g]_{z} \in \mathcal{S}_{z}^{j}$ with $i \neq j$ and any pair of unit vectors $\boldsymbol{l}, \boldsymbol{l}^{\prime}$,

$$
\begin{aligned}
R_{l}\left(\frac{\partial[f]_{z}}{\partial x}, \frac{\partial[f]_{z}}{\partial y}\right)>0 & \wedge R_{l^{\prime}}\left(\frac{\partial[g]_{z}}{\partial x}, \frac{\partial[g]_{z}}{\partial y}\right)>0 \\
& \Downarrow \\
R_{l}\left(\frac{\partial[f]_{z}}{\partial x}, \frac{\partial[f]_{z}}{\partial y}\right) & \neq R_{l^{\prime}}\left(\frac{\partial[g]_{z}}{\partial x}, \frac{\partial[g]_{z}}{\partial y}\right) .
\end{aligned}
$$

(B) For $[f]_{z} \in \mathcal{S}_{z}^{i}$ and $[g]_{z} \in \mathcal{S}_{z}^{j}$ with $i \neq j$ and any pair of unit vectors $\boldsymbol{l}, \boldsymbol{l}^{\prime}$ such that $\boldsymbol{l} \neq \boldsymbol{l}^{\prime}$, implication (17) holds.

The above conditions can alternatively be expressed as follows. A family $\left\{\mathcal{S}_{z}^{i}\right\}_{i \in I}$ of uniqueness classes satisfies (A) if any pair consisting of elements of two different building blocks of the form $\mathcal{S}_{z}^{i}(i \in I)$ generates images that never coincide. Similarly, a family of uniqueness classes satisfies (B) if any pair comprising elements of two different building blocks of this family generates images that coincide only when the corresponding illuminant vectors coincide. Note that condition (B) is less restrictive than condition (A). A moment's reflection shows that if a family $\left\{\mathcal{S}_{z}^{i}\right\}_{i \in I}$ of uniqueness classes fulfills either (A) or (B) (in either case (B) is satisfied), then $\bigcup_{i \in I} \mathcal{S}_{z}^{i}$ is a uniqueness class. Thus, if a family of uniqueness classes fulfills either of the above conditions, then a new larger uniqueness class can be formed by taking the sum of all elements of the family. As far as formation of uniqueness classes is concerned, the building blocks of a family that obeys (B) but not (A) fit with each other in a more subtle way than do the building blocks of a family satisfying (A). This is because condition (B) permits coincidence of images from distinct building blocks, whereas condition (A) does not.

By way of illustration, we now present two families of uniqueness classes, one of which satisfies (A), the other satisfying (B) but not (A). Both families will consist of singletons, and the corresponding sums will form uniqueness classes containing more than one element. The first family comprises germs some of whose representatives are hemispherical in shape. Let $z=(a, b)$ and, for each $r>0$, let $f_{r, z}$ be given by

$$
f_{r, z}(x, y)=\sqrt{r^{2}-(x-a)^{2}-(y-b)^{2}} \text {. }
$$


A straightforward calculation shows that, for each $r>0, D\left(f_{r, z}\right)(z)=r^{-2}$. Thus, by Theorem $1,\left\{\left[f_{r, z}\right]_{z}\right\}$ is a uniqueness class for each $r>0$. We have the following:

THEOREM 4. The family $\left\{\left\{\left[f_{r, z}\right]_{z}\right\}\right\}_{r>0}$ satisfies condition (A).

Proof. First note that if $\boldsymbol{l}=\left(l_{1}, l_{2}, l_{3}\right)$ is a unit vector, then

$$
\begin{aligned}
R_{l}\left(\frac{\partial f_{r, z}}{\partial x}, \frac{\partial f_{r, z}}{\partial y}\right) & (x, y) \\
& =\frac{1}{r}\left(l_{1}(x-a)+l_{2}(y-b)+l_{3} \sqrt{r^{2}-(x-a)^{2}-(y-b)^{2}}\right)
\end{aligned}
$$

Next observe that

$$
\frac{\partial^{2} R_{l}}{\partial x^{2}}\left(\frac{\partial f_{r, z}}{\partial x}, \frac{\partial f_{r, z}}{\partial y}\right)(z)=-\frac{l_{3}}{r^{2}}
$$

The theorem will be established once we show that if

$$
R_{l}\left(\frac{\partial f_{r, z}}{\partial x}, \frac{\partial f_{r, z}}{\partial y}\right)(x, y)=R_{l^{\prime}}\left(\frac{\partial f_{r^{\prime}, z}}{\partial x}, \frac{\partial f_{r^{\prime}, z}}{\partial y}\right)(x, y)>0
$$

for some positive numbers $r, r^{\prime}$, some unit vectors $l, l^{\prime}$, and all points $(x, y)$ in an open neighbourhood of $z$, then

$$
r=r^{\prime}
$$

Substituting $(a, b)$ for $(x, y)$ in $(20)$ and using $(18)$, we obtain

$$
l_{3}=l_{3}^{\prime}>0 \text {. }
$$

Similarly, differentiating twice at $z$ both sides of (20) with respect to $x$ and using (19), we find that

$$
\frac{l_{3}}{r^{2}}=\frac{l_{3}^{\prime}}{r^{\prime 2}}
$$

Combining this equality with (22) yields

$$
\frac{l_{3}}{r^{2}}=\frac{l_{3}}{r^{\prime 2}}
$$

Now, taking into account that $l_{3}>0$, we obtain (21).

As an immediate consequence, we find that, for each point $z,\left\{\left[f_{r, z}\right]_{z}: r>0\right\}$ is a uniqueness class at $z$. Hereafter, this class will be referred to as the hemispherical class at $z$.

To present the second family, define a function $f$ by setting

$$
f(x, y)=\sqrt{\left(\frac{(x-a)^{2}-(y-b)^{2}}{2}+1\right)^{2}+(x-a)^{2}(y-b)^{2}},
$$

where $a, b$ are real numbers. Letting $z=(a, b)$, we easily calculate that $D(f)(z)=-1$. By Theorem $1,\left\{[f]_{z}\right\}$ is a uniqueness class. Now fix $\alpha \in[0,2 \pi)$, and let $g_{\alpha}$ be given by (13). As we already know, $\left\{\left[g_{\alpha}\right]_{z}\right\}$ is a uniqueness class. We have the following: 
TheOREM 5. The family $\left\{\left\{[f]_{z}\right\},\left\{\left[g_{\alpha}\right]_{z}\right\}\right\}$ satisfies condition (B) but does not satisfy condition (A). More specifically, the image of $[f]_{z}$ corresponding to the illuminant vector $\boldsymbol{e}$ is identical with the image of $\left[g_{\alpha}\right]_{z}$ corresponding to the same illuminant vector $\boldsymbol{e}$. Furthermore, if any image of $[f]_{z}$ coincides with an image of $\left[g_{\alpha}\right]_{z}$, then the corresponding illuminant vectors also coincide and are equal to $\boldsymbol{e}$.

The proof will be based on the following result:

Lemma 1 . Let $l_{1}, l_{2}, l_{3}, A, B, C$ be real numbers such that

$$
l_{1}\left(x^{2}+y^{2}+2\right) x+l_{2}\left(x^{2}+y^{2}-2\right) y=\left(l_{3}-A x-B y-C\right) \sqrt{\left(x^{2}-y^{2}+2\right)^{2}+4 x^{2} y^{2}}
$$

for $(x, y)$ in an open neighbourhood of $(0,0)$. Then $l_{1}=l_{2}=A=B=0$ and $l_{3}=C$.

Proof. First, substituting 0 for $x$ and $y$ in (24), we find that $l_{3}=C$. Next, substituting 0 for $y$ and letting $x$ vary, we see that

$$
l_{1}\left(x^{2}+2\right) x=-A x\left(x^{2}+2\right),
$$

whence $l_{1}=-A$. Similarly, substituting 0 for $x$ and letting $y$ vary, we see that for $y$ sufficiently small

$$
l_{2}\left(y^{2}-2\right) y=-B y\left(2-y^{2}\right),
$$

whence $l_{2}=B$. We thus see that, for $(x, y)$ in an open neighbourhood of $(0,0)$,

$$
l_{1}\left(x^{2}+y^{2}+2\right) x+l_{2}\left(x^{2}+y^{2}-2\right) y=\left(l_{1} x-l_{2} y\right) \sqrt{\left(x^{2}-y^{2}+2\right)^{2}+4 x^{2} y^{2}}
$$

and further

$$
\left(l_{1}\left(x^{2}+y^{2}+2\right) x+l_{2}\left(x^{2}+y^{2}-2\right) y\right)^{2}=\left(l_{1} x-l_{2} y\right)^{2}\left(\left(x^{2}-y^{2}+2\right)^{2}+4 x^{2} y^{2}\right) .
$$

Since both sides of the last equation are polynomials, the equality holds for all $x$ and $y$. Substituting $l_{2}$ for $x$ and $l_{1}$ for $y$, we see that

$$
\left(l_{1} l_{2}\left(l_{1}^{2}+l_{2}^{2}\right)\right)^{2}=0,
$$

whence either $l_{1}=0$ or $l_{2}=0$. We now show that in fact

$$
l_{1}=l_{2}=0 .
$$

To this end, suppose that $l_{1}=0$ and $l_{2} \neq 0$. Then, on account of (25),

$$
\left(l_{2}\left(x^{2}+y^{2}-2\right) y\right)^{2}=l_{2}^{2} y^{2}\left(\left(x^{2}-y^{2}+2\right)^{2}+4 x^{2} y^{2}\right)
$$

whence

$$
\left(x^{2}+y^{2}-2\right)^{2}=\left(x^{2}-y^{2}+2\right)^{2}+4 x^{2} y^{2},
$$

which is not valid for, say, $x=y=1$. Analogously, the conjunction of the relations $l_{2}=0$ and $l_{1} \neq 0$ leads to a contradiction. Thus (26) is established, and the lemma follows. 
Proof of Theorem 5. Without loss of generality, we may assume that $a=b=0$. A straightforward calculation shows that

$$
\begin{aligned}
\frac{\partial f}{\partial x}(x, y) & =\frac{\left(x^{2}+y^{2}+2\right) x}{\sqrt{\left(x^{2}-y^{2}+2\right)^{2}+4 x^{2} y^{2}}}, \\
\frac{\partial f}{\partial y}(x, y) & =\frac{\left(x^{2}+y^{2}-2\right) y}{\sqrt{\left(x^{2}-y^{2}+2\right)^{2}+4 x^{2} y^{2}}}, \\
\frac{\partial g_{\alpha}}{\partial x}(x, y) & =x \cos \alpha+y \sin \alpha, \\
\frac{\partial g_{\alpha}}{\partial y}(x, y) & =x \sin \alpha-y \cos \alpha .
\end{aligned}
$$

Hence

$$
\left(\frac{\partial f}{\partial x}(x, y)\right)^{2}+\left(\frac{\partial f}{\partial y}(x, y)\right)^{2}=\left(\frac{\partial g_{\alpha}}{\partial x}(x, y)\right)^{2}+\left(\frac{\partial g_{\alpha}}{\partial y}(x, y)\right)^{2}=x^{2}+y^{2}
$$

Taking into account that

$$
R_{e}(p, q)=\frac{1}{\sqrt{p^{2}+q^{2}+1}}
$$

we see that the image of $[f]_{z}$ corresponding to the illuminant vector $e$ is identical with the image of $\left[g_{\alpha}\right]_{z}$ corresponding to the same illuminant vector $\boldsymbol{e}$.

Suppose now that, for $(x, y)$ in an open neighbourhood of $(0,0)$,

$$
R_{l}\left(\frac{\partial f}{\partial x}, \frac{\partial f}{\partial y}\right)(x, y)=R_{l^{\prime}}\left(\frac{\partial g_{\alpha}}{\partial x}, \frac{\partial g_{\alpha}}{\partial y}\right)(x, y)
$$

and that both sides of the equality are positive. Then, in view of (27) and (28), the above equality can be rewritten, up to a positive factor, as

$$
\begin{aligned}
& -\frac{l_{1}\left(x^{2}+y^{2}+2\right) x+l_{2}\left(x^{2}+y^{2}-2\right) y}{\sqrt{\left(x^{2}-y^{2}+2\right)^{2}+4 x^{2} y^{2}}}+l_{3} \\
& \qquad=-\left(l_{1}^{\prime} \cos \alpha+l_{2}^{\prime} \sin \alpha\right) x-\left(l_{1}^{\prime} \sin \alpha-l_{2}^{\prime} \cos \alpha\right) y+l_{3}^{\prime} .
\end{aligned}
$$

Substituting 0 for $x$ and $y$, we see that $l_{3}=l_{3}^{\prime}>0$. In view of Lemma $1, l_{1}=l_{2}=0$ and $l_{1}^{\prime} \cos \alpha+l_{2}^{\prime} \sin \alpha=l_{1}^{\prime} \sin \alpha-l_{2}^{\prime} \cos \alpha^{\prime}=0$. But the latter set of equalities immediately implies that $l_{1}^{\prime}=l_{2}^{\prime}=0$. Now it is clear that $l=l^{\prime}=\boldsymbol{e}$.

As an immediate consequence, we infer that $\left\{[f]_{z},\left[g_{\alpha}\right]_{z}\right\}$ is a uniqueness class.

Considering various candidate blocks of uniqueness classes and selecting those whose composites satisfy either (A) or (B), we can successively build larger and larger uniqueness classes. A natural question arises as to whether this process must ever halt. The answer is in the affirmative. Before we elaborate on that, we present necessary settheoretic prerequisites (see [7, p. 12]).

Let $S$ be a set. A partial ordering of $S$ is a relation, written $s \prec t$, amongst some pairs of elements of $S$, having the following properties:

(i) $s \prec s$;

(ii) $s \prec t \wedge t \prec u \Rightarrow s \prec u$;

(iii) $s \prec t \wedge t \prec s \Rightarrow s=t$. 
A partially ordered set $S$ is linearly ordered if given any $s, t \in S$ we have either $s \prec t$ or $t \prec s$. Let $S$ be a partially ordered set. A maximal element of $S$ is an element $m$ such that if $s \in S$ and $m \prec s$, then $s=m$. The greatest element of $S$ is an element $g$ such that $s \prec g$ for all $s \in S$. Note that a maximal element need not be the greatest element. There may be many maximal elements in $S$, whereas if a greatest element exists, then it is unique. Let $T$ be a subset of $S$. An upper bound of $T$ in $S$ is an element $t \in S$ such that $s \prec t$ for all $s \in T$. The least upper bound of $T$ in $S$ is an upper bound $t$ such that, if $u$ is another upper bound, then $t \prec u$. If a least upper bound of $T$ exists, then it is unique.

Observe that the family $\Gamma_{z}$ of all uniqueness classes at a given point $z$ can be partially ordered as follows: Given $\mathcal{S}_{z}^{\prime}, \mathcal{S}_{z}^{\prime \prime} \in \Gamma_{z}$, we declare $\mathcal{S}_{z}^{\prime \prime}$ to be a successor of $\mathcal{S}_{z}^{\prime}$ and write $\mathcal{S}_{z}^{\prime} \prec \mathcal{S}_{z}^{\prime \prime}$ whenever $\mathcal{S}_{z}^{\prime} \subset \mathcal{S}_{z}^{\prime \prime}$. A fundamental property of this ordering is stated in the following:

THEOREM 6. For each point $z$ in the $(x, y)$-plane, every uniqueness class at $z$ is contained in a maximal uniqueness class at $z$ with respect to $\prec$.

Proof. In view of a set-theoretic result known as the Kuratowski-Zorn lemma (see [7, Theorem 16]), it is sufficient to verify that every linearly ordered subset of $\boldsymbol{\Gamma}_{z}$ has an upper bound. Let $\boldsymbol{\Psi}$ be a linearly ordered subset of $\boldsymbol{\Gamma}_{z}$. We shall show that $\bigcup_{\mathcal{S}_{z} \in \Psi} \mathcal{S}_{z}$ is an upper bound (even the least, upper bound) for $\boldsymbol{\Psi}$ in $\boldsymbol{\Gamma}_{z}$. To this end, we shall demonstrate that $\bigcup_{\mathcal{S}_{z} \in \Psi} \mathcal{S}_{z}$ is a uniqueness class. Once this is done, the verification that $\bigcup_{\mathcal{S}_{z} \in \Psi} \mathcal{S}_{z}$ is the least upper bound for $\boldsymbol{\Psi}$ is straightforward. Suppose that $[f]_{z}$ and $[g]$ are two members of $\bigcup_{\mathcal{S}_{z} \in \Psi} \mathcal{S}_{z}$. Then $[f]_{z} \in \mathcal{S}_{z}^{\prime}$ and $[g]_{z} \in \mathcal{S}_{z}^{\prime \prime}$ for some $\mathcal{S}_{z}^{\prime}, \mathcal{S}_{z}^{\prime \prime} \in \Psi$. Since $\boldsymbol{\Psi}$ is linearly ordered, we have either $\mathcal{S}_{z}^{\prime} \subset \mathcal{S}_{z}^{\prime \prime}$ or $\mathcal{S}_{z}^{\prime \prime} \subset \mathcal{S}_{z}^{\prime}$. Without loss of generality, we may assume that the first containment takes place. Then both $[f]_{z}$ and $[g]_{z}$ belong to $\mathcal{S}_{z}^{\prime \prime}$, and, since $\mathcal{S}_{z}^{\prime \prime}$ is a uniqueness class, implication (3) holds, showing that $\bigcup_{\mathcal{S}_{z} \in \Psi} \mathcal{S}_{z}$ is a uniqueness class.

A given uniqueness class may be part of many maximal uniqueness classes. To see this, for each $\alpha \in[0,2 \pi)$, let $g_{\alpha}$ be given by (13), and let $f$ be given by (23). By Theorem 5 , for each $\alpha \in[0,2 \pi)$, the set $\left\{[f]_{z},\left[g_{\alpha}\right]_{z}\right\}$ is a uniqueness class. Extend $\left\{[f]_{z},\left[g_{\alpha}\right]_{z}\right\}$ to a maximal uniqueness class $\mathcal{M}_{z}^{(\alpha)}$. We claim that $\mathcal{M}_{z}^{(\alpha)}$ is different from $\mathcal{M}_{z}^{(\beta)}$ whenever $\alpha \neq \beta(\alpha, \beta \in[0,2 \pi))$. Indeed, $\mathcal{M}_{z}^{(\alpha)}=\mathcal{M}_{z}^{(\beta)}$ for some $\alpha, \beta \in[0,2 \pi)$ with $\alpha \neq \beta$ would imply that $g_{\alpha}$ and $g_{\beta}$ belong to the same uniqueness class, namely $\mathcal{M}_{z}^{(\alpha)}$, contradicting Theorem 3. Notice that all the $\mathcal{M}_{z}^{(\alpha)}$ contain the uniqueness class consisting of the germ $[f]_{z}$.

The larger a uniqueness class, the larger will be the corresponding image class that serves as the domain of applicability of the associated light-source-direction estimator. Thus a light-source-direction estimator based on a maximal uniqueness class is in a sense optimal. The most desirable candidate for a maximal uniqueness class at a given point $z$ is of course $\mathcal{C}_{z}^{2}$. But $\mathcal{C}_{z}^{2}$ is not even a uniqueness class. Indeed, if $\mathcal{C}_{z}^{2}$ were a uniqueness class, then it would be the greatest element of $\Gamma_{z}$, and hence there would be only one maximal uniqueness class at $z$, namely $\mathcal{C}_{z}^{2}$ itself. This, however, is impossible in view of the previous paragraph. 
We close this section by remarking that finding an analytical formula for a light-sourcedirection estimator associated with a particular uniqueness class is a separate problem. For a hemispherical class such a formula was derived in [2].

4. Fundamental ambiguity. As shown in the previous section, there are many uniqueness classes at any point in the $(x, y)$-plane. This, however, does not preclude the existence of images $E$ with the following property: For some point $z$ in an image domain, there exists a single uniqueness class $\mathcal{S}_{z}$ such that $[E]_{z}$ is a member of the corresponding image class $\mathcal{E}_{\mathcal{S}_{z}}$. Light-source-direction estimation would be greatly facilitated if a given image $E$ enjoyed the above property. For if $z$ were a point in the image domain to which the property applies and $\mathcal{S}_{z}$ were the corresponding image class, then $\Lambda_{S_{z}}\left([E]_{z}\right)$ would be the sought-after illuminant vector. The main purpose of this section is to show that typical images do not have the above property. Thus in general light-source-direction estimation, based on local shading data, is hampered by the problem of which uniqueness class to use.

We begin by establishing an auxiliary result.

For a unit vector $l$, an open subset $U$ of the $(x, y)$-plane, and a positive $C^{2}$ function $E$ on $U$, set

$$
F_{l}(x, y, p, q)=E(x, y) \sqrt{p^{2}+q^{2}+1}+l_{1} p+l_{2} q-l_{3} .
$$

Clearly, Eq. (1) is equivalent to

$$
F_{l}=\left(x, y, \frac{\partial f}{\partial x}, \frac{\partial f}{\partial y}\right)=0 .
$$

A point $z=(a, b) \in U$ is said to be singular if, for some real numbers $p, q$,

$$
F_{l}(a, b, p, q)=0
$$

alongside

$$
\frac{\partial F_{l}}{\partial p}(a, b, p, q)=0 \quad \text { and } \quad \frac{\partial F_{l}}{\partial q}(a, b, p, q)=0 .
$$

We have the following characterisation of singular points:

TheOREM 7. With the notation as above, if $z \in U$ is a singular point, then $E(z)=1$.

Proof. Let $z \in U$ be a singular point such that (30) and (31) hold for some real numbers $p, q$. Then, by (31),

$$
\frac{E(z) p}{\sqrt{p^{2}+q^{2}+1}}+l_{1}=\frac{E(z) q}{\sqrt{p^{2}+q^{2}+1}}+l_{2}=0 .
$$

Hence, by (30),

$$
p\left(l_{3}-l_{1} p-l_{2} q\right)+l_{1}\left(p^{2}+q^{2}+1\right)=q\left(l_{3}-l_{1} p-l_{2} q\right)+l_{2}\left(p^{2}+q^{2}+1\right)=0
$$

and so

$$
\begin{aligned}
-\left(1+q^{2}\right) l_{1}+p q l_{2} & =p l_{3}, \\
p q l_{1}-\left(1+p^{2}\right) l_{2} & =q l_{3} .
\end{aligned}
$$


Solving this system of equations for $l_{1}$ and $l_{2}$ yields

$$
l_{1}=-p l_{3} \quad \text { and } \quad l_{2}=-q l_{3} .
$$

These equalities together with (30) show that

$$
E(z)=l_{3} \sqrt{p^{2}+q^{2}+1} .
$$

On the other hand, taking into account (32) and the fact that $l$ has unit length, we see that

$$
l_{3}^{2}\left(p^{2}+q^{2}+1\right)=l_{1}^{2}+l_{2}^{2}+l_{3}^{2}=1 .
$$

The latter relation together with (33) and the fact that $E$ is positive implies that $E(z)=$ 1. The result follows.

THEOREM 8. Let $z$ be a point in the $(x, y)$-plane, let $U$ be an open neighbourhood of $z$, and let $E$ be a positive $C^{2}$ function on $U$ such that

$$
E(z)<1
$$

and

$$
\left(\frac{\partial E}{\partial x}(z)\right)^{2}+\left(\frac{\partial E}{\partial y}(z)\right)^{2}>0 .
$$

Then, for each unit vector $l=\left(l_{1}, l_{2}, l_{3}\right)$ with $l_{3}>0$, there exists an open neighbourhood $V \subset U$ of $z$ and a $C^{2}$ function $f$ on $V$ satisfying (1) and (5).

Proof. Observe first that, since $E(z)>0$ and $l_{3}>0$, there exist real numbers $m, n$ such that $R_{l}(m, n)=E(z)$, or equivalently,

$$
F_{l}(a, b, m, n)=0,
$$

where $z=(a, b)$. In fact, we can always represent $l$ in the form

$$
l=l_{3} e+\mu f
$$

where $f$ is a unit vector orthogonal to $e$, and $\mu$ is a real number such that $l_{3}^{2}+\mu^{2}=1$. For if $l_{3}=1$, then $l=e$ for whatever choice of $f$ as a unit vector orthogonal to $e$, and if $l_{3}<1$, then it suffices to take

$$
\frac{l-l_{3} e}{\left\|l-l_{3} e\right\|}
$$

for $f$; here $\|\cdot\|$ denotes the Euclidean norm derived from the usual scalar product $\langle\cdot \mid \cdot\rangle$. Let $\alpha=\arccos E(z)$ and

$$
\begin{aligned}
\boldsymbol{n}_{\alpha} & =\left(l_{3} \cos \alpha-\mu \sin \alpha\right) \boldsymbol{e}+\left(l_{3} \sin \alpha+\mu \cos \alpha\right) f \\
\boldsymbol{n}_{-\alpha} & =\left(l_{3} \cos \alpha+\mu \sin \alpha\right) \boldsymbol{e}+\left(-l_{3} \sin \alpha+\mu \cos \alpha\right) \boldsymbol{f} .
\end{aligned}
$$

Using the above expansions of $\boldsymbol{l}, \boldsymbol{n}_{\alpha}$, and $\boldsymbol{n}_{-\alpha}$ in the orthogonal basis $\{\boldsymbol{e}, \boldsymbol{f}\}$, it is easy to verify that $\boldsymbol{n}_{\alpha}$ and $\boldsymbol{n}_{-\alpha}$ are unit vectors such that

$$
\left\langle\boldsymbol{n}_{\alpha} \mid \boldsymbol{l}\right\rangle=\left\langle\boldsymbol{n}_{-\alpha} \mid \boldsymbol{l}\right\rangle=\cos \alpha=E(z) .
$$


Since the sum of the $\boldsymbol{e}$-components of $\boldsymbol{n}_{\alpha}$ and $n_{-\alpha}$ equals $2 l_{3} E(z)$, at least one of the vectors $\boldsymbol{n}_{\alpha}$ and $\boldsymbol{n}_{-\alpha}$, say $\boldsymbol{n}_{\alpha}$, has a positive $\boldsymbol{e}$-component. Now, letting $\boldsymbol{n}_{\alpha}=$ $\left(n_{1}^{(\alpha)}, n_{2}^{(\alpha)}, n_{3}^{(\alpha)}\right)$, set

$$
m=-\frac{n_{1}^{(\alpha)}}{n_{3}^{(\alpha)}} \quad \text { and } \quad n=-\frac{n_{2}^{(\alpha)}}{n_{3}^{(\alpha)}} .
$$

It is readily verified that $m$ and $n$ satisfy (36).

In view of Theorem 7 and (34), either

$$
\frac{\partial F_{l}}{\partial p}(a, b, m, n) \neq 0
$$

or

$$
\frac{\partial F_{l}}{\partial q}(a, b, m, n) \neq 0
$$

Without loss of generality, we may suppose that the latter inequality holds. Let $P$ be any real $C^{1}$ function on $U$ such that $P(z)=m$ and

$$
\left|\begin{array}{ll}
\frac{\partial P}{\partial x}(z) & \frac{\partial P}{\partial y}(z) \\
\frac{\partial E}{\partial x}(z) & \frac{\partial E}{\partial y}(z)
\end{array}\right| \neq 0
$$

for each $z \in U$. For example, we may assume that

$$
P(x, y)=m+\frac{\partial E}{\partial y}(z)(x-a)+\frac{\partial E}{\partial x}(z)(b-y)
$$

in which case

$$
\left|\begin{array}{ll}
\frac{\partial P}{\partial x}(z) & \frac{\partial P}{\partial y}(z) \\
\frac{\partial E}{\partial x}(z) & \frac{\partial E}{\partial y}(z)
\end{array}\right|=\left(\frac{\partial E}{\partial x}(z)\right)^{2}+\left(\frac{\partial E}{\partial y}(z)\right)^{2}
$$

is positive by virtue of (35). In view of the implicit function theorem, (36), and (37), there exists an open neighbourhood $V \subset U$ of $z$ and a unique real $C^{1}$ function $Q$ on $V$ such that $Q(z)=n$ and

$$
F_{l}(x, y, P(x, y), Q(x, y))=0
$$

for each $(x, y) \in V$. In view of (37), we may assume, shrinking $V$ if necessary, that

$$
\frac{\partial F_{l}}{\partial q}(x, y, P(x, y), Q(x, y)) \neq 0
$$

for each $(x, y) \in V$. Differentiating both sides of (39) with respect to $x$ and $y$, we obtain

$$
\begin{aligned}
& \frac{\partial F_{l}}{\partial x}+\frac{\partial F_{l}}{\partial p} \frac{\partial P}{\partial x}+\frac{\partial F_{l}}{\partial q} \frac{\partial Q}{\partial x}=0 \\
& \frac{\partial F_{l}}{\partial y}+\frac{\partial F_{l}}{\partial p} \frac{\partial P}{\partial y}+\frac{\partial F_{l}}{\partial q} \frac{\partial Q}{\partial y}=0 .
\end{aligned}
$$


Hence, by (40),

$$
\begin{aligned}
& \frac{\partial Q}{\partial x}=-\frac{\frac{\partial F_{l}}{\partial x}+\frac{\partial F_{l}}{\partial p} \frac{\partial P}{\partial x}}{\frac{\partial F_{l}}{\partial q}} \\
& \frac{\partial Q}{\partial y}=-\frac{\frac{\partial F_{l}}{\partial y}+\frac{\partial F_{l}}{\partial p} \frac{\partial P}{\partial y}}{\frac{\partial F_{l}}{\partial q}}
\end{aligned}
$$

and further

$$
\begin{aligned}
\left|\begin{array}{ll}
\frac{\partial P}{\partial x} & \frac{\partial P}{\partial y} \\
\frac{\partial Q}{\partial x} & \frac{\partial Q}{\partial y}
\end{array}\right| & =-\frac{1}{\left(\frac{\partial F_{l}}{\partial q}\right)^{2}}\left|\begin{array}{ccc}
\frac{\partial P}{\partial x} & \frac{\partial P}{\partial y} \\
\frac{\partial F_{l}}{\partial x}+\frac{\partial F_{l}}{\partial p} \frac{\partial P}{\partial x} & \frac{\partial F_{l}}{\partial y}+\frac{\partial F_{l}}{\partial p} \frac{\partial P}{\partial y}
\end{array}\right| \\
& =-\frac{1}{\left(\frac{\partial F_{l}}{\partial q}\right)^{2}}\left|\begin{array}{cc}
\frac{\partial P}{\partial x} & \frac{\partial P}{\partial y} \\
\frac{\partial F_{l}}{\partial x} & \frac{\partial F_{l}}{\partial y}
\end{array}\right| .
\end{aligned}
$$

Taking into account that

$$
\begin{aligned}
& \frac{\partial F_{l}}{\partial x}(x, y, p, q)=\sqrt{p^{2}+q^{2}+1} \frac{\partial E}{\partial x}(x, y), \\
& \frac{\partial F_{l}}{\partial y}(x, y, p, q)=\sqrt{p^{2}+q^{2}+1} \frac{\partial E}{\partial y}(x, y),
\end{aligned}
$$

we can rewrite $(38)$ as

$$
\left|\begin{array}{cc}
\frac{\partial P}{\partial x}(z) & \frac{\partial P}{\partial y}(z) \\
\frac{\partial F_{l}}{\partial x}(a, b, m, n) & \frac{\partial F_{l}}{\partial y}(a, b, m, n)
\end{array}\right| \neq 0 .
$$

Now this inequality together with (41) yields

$$
\left|\begin{array}{ll}
\frac{\partial P}{\partial x}(z) & \frac{\partial P}{\partial y}(z) \\
\frac{\partial Q}{\partial x}(z) & \frac{\partial Q}{\partial y}(z)
\end{array}\right| \neq 0
$$

We claim that

$$
\left|\begin{array}{ll}
\frac{\partial P}{\partial x}(z) & \frac{\partial F_{l}}{\partial x}(a, b, m, n) \\
\frac{\partial Q}{\partial x}(z) & \frac{\partial F_{l}}{\partial y}(a, b, m, n)
\end{array}\right|^{2}+\left|\begin{array}{ll}
\frac{\partial P}{\partial y}(z) & \frac{\partial F_{l}}{\partial x}(a, b, m, n) \\
\frac{\partial Q}{\partial y}(z) & \frac{\partial F_{l}}{\partial y}(a, b, m, n)
\end{array}\right|^{2}>0 .
$$

Indeed, in view of (35) and (42), the vector

$$
\boldsymbol{f}=\left(\frac{\partial F_{l}}{\partial x}(a, b, m, n), \frac{\partial F_{l}}{\partial y}(a, b, m, n)\right)
$$

is nonzero. If the left-hand side of (44) were zero, then each of the vectors

$$
\begin{aligned}
& \boldsymbol{p}=\left(\frac{\partial P}{\partial x}(z), \frac{\partial Q}{\partial x}(z)\right) \\
& \boldsymbol{q}=\left(\frac{\partial P}{\partial y}(z), \frac{\partial Q}{\partial y}(z)\right)
\end{aligned}
$$

would be a scalar multiple of $\boldsymbol{f}$, and so $\boldsymbol{p}$ and $\boldsymbol{q}$ would be linearly dependent, contrary to $(43)$.

We now pause for a while to establish the following assertion: If $\boldsymbol{a}$ and $\boldsymbol{b}$ are nonzero vectors, then there exists a vector $v$ such that $\langle\boldsymbol{a} \mid \boldsymbol{v}\rangle \neq 0$ and $\langle\boldsymbol{b} \mid \boldsymbol{v}\rangle \neq 0$. Indeed, if $\boldsymbol{a}$ 
and $\boldsymbol{b}$ are linearly dependent, then it suffices to take $\boldsymbol{a}$ for $\boldsymbol{v}$. If $\boldsymbol{a}$ and $\boldsymbol{b}$ are linearly independent, then an application of the Gram-Schmidt orthogonalisation technique gives

$$
\begin{aligned}
& \boldsymbol{a}=a_{11} \boldsymbol{e}_{1}, \\
& \boldsymbol{b}=a_{21} \boldsymbol{e}_{1}+a_{22} \boldsymbol{e}_{2}
\end{aligned}
$$

for some orthogonal basis $\boldsymbol{e}_{1}, \boldsymbol{e}_{2}$, where $a_{i j}(i, j \in\{1,2\})$ are real numbers such that

$$
\left|\begin{array}{cc}
a_{11} & 0 \\
a_{21} & a_{22}
\end{array}\right|=a_{11} a_{22} \neq 0,
$$

or, equivalently, such that $a_{11} \neq 0$ and $a_{22} \neq 0$; now, if we let

$$
\boldsymbol{v}=\frac{1}{a_{11}} e_{1}+\left(\frac{1}{a_{22}}-\frac{a_{21}}{a_{11} a_{22}}\right) e_{2},
$$

then $\langle\boldsymbol{a} \mid \boldsymbol{v}\rangle=\langle\boldsymbol{b} \mid \boldsymbol{v}\rangle=1$.

Using the above assertion together with (37) and (44), we see that there exists $v=$ $\left(v_{1}, v_{2}\right)$ such that

$$
\left|\begin{array}{cc}
v_{1} & v_{2} \\
\frac{\partial F_{l}}{\partial p}(a, b, m, n) & \frac{\partial F_{l}}{\partial q}(a, b, m, n)
\end{array}\right| \neq 0
$$

and

$$
v_{1}\left|\begin{array}{ll}
\frac{\partial P}{\partial x}(z) & \frac{\partial F_{l}}{\partial x}(a, b, m, n) \\
\frac{\partial Q}{\partial x}(z) & \frac{\partial F_{l}}{\partial y}(a, b, m, n)
\end{array}\right|+v_{2}\left|\begin{array}{ll}
\frac{\partial P}{\partial y}(z) & \frac{\partial F_{l}}{\partial x}(a, b, m, n) \\
\frac{\partial Q}{\partial y}(z) & \frac{\partial F_{l}}{\partial y}(a, b, m, n)
\end{array}\right| \neq 0 .
$$

Let $I$ be an open interval containing 0 , and let $I \ni s \mapsto(x(s), y(s)) \in V$ be a $C^{2}$ function such that

$$
\begin{array}{ll}
x(0)=a, & y(0)=b, \\
\dot{x}(0)=v_{1}, & \dot{y}(0)=v_{2} .
\end{array}
$$

For each $s \in I$, set

$$
\begin{aligned}
& p(s)=P(x(s), y(s)), \\
& q(s)=Q(x(s), y(s)) .
\end{aligned}
$$

The function $I \ni s \mapsto(x(s), y(s), p(s), q(s)) \in V \times \mathbb{R}^{2}$ furnishes initial data for the Cauchy problem of finding a $C^{2}$ solution $f$ to (29) satisfying

$$
\begin{aligned}
& \frac{\partial f}{\partial x}(x(s), y(s))=p(s), \\
& \frac{\partial f}{\partial y}(x(s), y(s))=q(s) .
\end{aligned}
$$

Now (45) means that the initial data are non-characteristic at $s=0$. By a fundamental theorem concerning solvability of the Cauchy problem for first-order partial differential equations (cf. [3, Chapter VI, Theorem 9.1]), there exists an open neighbourhood $W \subset V$ of $z$, an open interval $J \subset I$ containing 0 , and a $C^{2}$ function $f$ on $W$ such that (29) holds in $W$ and (49) is valid for all $s \in J$. Clearly, $f$ is also a $C^{2}$ solution to (1) in $W$. To end the proof, it suffices to show that $f$ satisfies (5). 
Differentiating both sides of (29) at $z$ with respect to $x$ and $y$, we obtain

$$
\begin{aligned}
& \frac{\partial F_{l}}{\partial x}(z)+\frac{\partial F_{l}}{\partial p}(a, b, m, n) \frac{\partial^{2} f}{\partial x^{2}}(z)+\frac{\partial F_{l}}{\partial q}(a, b, m, n) \frac{\partial^{2} f}{\partial x \partial y}(z)=0, \\
& \frac{\partial F_{l}}{\partial y}(z)+\frac{\partial F_{l}}{\partial p}(a, b, m, n) \frac{\partial^{2} f}{\partial x \partial y}(z)+\frac{\partial F_{l}}{\partial q}(a, b, m, n) \frac{\partial^{2} f}{\partial y^{2}}(z)=0 .
\end{aligned}
$$

On the other hand, differentiating both sides of (49) at 0 with respect to $s$, and using (47b) and (48), we get

$$
\begin{aligned}
& v_{1} \frac{\partial^{2} f}{\partial x^{2}}(z)+v_{2} \frac{\partial^{2} f}{\partial x \partial y}(z)=v_{1} \frac{\partial P}{\partial x}(z)+v_{2} \frac{\partial P}{\partial y}(z), \\
& v_{1} \frac{\partial^{2} f}{\partial x \partial y}(z)+v_{2} \frac{\partial^{2} f}{\partial y^{2}}(z)=v_{1} \frac{\partial Q}{\partial x}(z)+v_{2} \frac{\partial Q}{\partial y}(z) .
\end{aligned}
$$

These two sets of equations can be rewritten in the matrix form

$$
\begin{aligned}
{\left[\begin{array}{ll}
\frac{\partial^{2} f}{\partial x^{2}}(z) & \frac{\partial^{2} f}{\partial x \partial y}(z) \\
\frac{\partial^{2} f}{\partial x \partial y}(z) & \frac{\partial^{2} f}{\partial y^{2}}(z)
\end{array}\right] } & \cdot\left[\begin{array}{ll}
\frac{\partial F_{l}}{\partial p}(a, b, m, n) & v_{1} \\
\frac{\partial F_{l}}{\partial q}(a, b, m, n) & v_{2}
\end{array}\right] \\
& =\left[\begin{array}{ll}
-\frac{\partial F_{l}}{\partial x}(a, b, m, n) & v_{1} \frac{\partial P}{\partial x}(z)+v_{2} \frac{\partial P}{\partial y}(z) \\
-\frac{\partial F_{l}}{\partial y}(a, b, m, n) & v_{1} \frac{\partial Q}{\partial x}(z)+v_{2} \frac{\partial Q}{\partial y}(z)
\end{array}\right]
\end{aligned}
$$

Hence

$$
\begin{aligned}
& \left|\begin{array}{ll}
\frac{\partial^{2} f}{\partial x^{2}}(z) & \frac{\partial^{2} f}{\partial x \partial y}(z) \\
\frac{\partial^{2} f}{\partial x \partial y}(z) & \frac{\partial^{2} f}{\partial y^{2}}(z)
\end{array}\right| \cdot\left|\begin{array}{ll}
\frac{\partial F_{l}}{\partial p}(a, b, m, n) & v_{1} \\
\frac{\partial F_{l}}{\partial q}(a, b, m, n) & v_{2}
\end{array}\right| \\
& \quad=-\left|\begin{array}{ll}
\frac{\partial F_{l}}{\partial x}(a, b, m, n) & v_{1} \frac{\partial P}{\partial x}(z)+v_{2} \frac{\partial P}{\partial y}(z) \\
\frac{\partial F_{l}}{\partial y}(a, b, m, n) & v_{1} \frac{\partial Q}{\partial x}(z)+v_{2} \frac{\partial Q}{\partial y}(z)
\end{array}\right| \\
& \quad=v_{1}\left|\begin{array}{ll}
\frac{\partial P}{\partial x}(z) & \frac{\partial F_{l}}{\partial x}(a, b, m, n) \\
\frac{\partial Q}{\partial x}(z) & \frac{\partial F_{l}}{\partial y}(a, b, m, n)
\end{array}\right|+v_{2}\left|\begin{array}{ll}
\frac{\partial P}{\partial y}(z) & \frac{\partial F_{l}}{\partial x}(a, b, m, n) \\
\frac{\partial Q}{\partial y}(z) & \frac{\partial F_{l}}{\partial y}(a, b, m, n)
\end{array}\right| .
\end{aligned}
$$

Combining the latter equality with (46), we obtain (5).

We are now in a position to state the main result of this section:

THEOREM 9. Let $z$ be a point in the $(x, y)$-plane, and let $[E]_{z} \in \mathcal{C}_{z}^{2}$ be such that $0<$ $[E]_{z}(z)<1$ and

$$
\left(\frac{\partial[E]_{z}}{\partial x}(z)\right)^{2}+\left(\frac{\partial[E]_{z}}{\partial y}(z)\right)^{2}>0 .
$$

Then there are infinitely many pairwise distinct maximal uniqueness classes at $z$ whose associated shape classes contain $[E]_{z}$.

Proof. By Theorem 8 , for each unit vector $l=\left(l_{1}, l_{2}, l_{3}\right)$ with $l_{3}>0$, there exists a solution $f_{l}$ satisfying (1) and (5). By Theorem $1,\left\{\left[f_{l}\right]_{z}\right\}$ is a uniqueness class. Extend $\left\{\left[f_{l}\right]_{z}\right\}$ arbitrarily to a maximal uniqueness class $\mathcal{M}_{z}^{(l)}$. Clearly, the image of $\left[f_{l}\right]_{z}$ from the direction $l$ is $[E]_{z}$, and therefore $[E]_{z} \in \mathcal{E}_{\mathcal{M}_{z}^{(l)}}$. Now, if $l, l^{\prime}$ are two distinct unit vectors with $l_{3}>0$ and $l_{3}^{\prime}>0$, respectively, then $\left[f_{l}\right]_{z}$ and $\left[f_{l^{\prime}}\right]_{z}$ cannot belong to a common 
uniqueness class (for (3) is violated if $f$ and $g$ are replaced by $f_{l}$ and $f_{l^{\prime}}$, respectively), and hence $\mathcal{M}_{z}^{(l)} \neq \mathcal{M}_{z}^{\left(l^{\prime}\right)}$. The theorem follows.

\section{REFERENCES}

[1] M. J. Brooks, W. Chojnacki, and R. Kozera, Impossible and ambiguous shading patterns, International Journal of Computer Vision 7, 119-126 (1992)

[2] W. Chojnacki, M. J. Brooks, and D. Gibbins, Revisiting Pentland's estimator of light source direction, Journal of the Optical Society of America A 11, 118-124 (1994)

[3] P. Hartman, Ordinary Differential Equations, Birkhäuser, Boston, 1982

[4] B. K. P. Horn, Obtaining shape from shading information, The Psychology of Computer Vision (P. H. Winston, ed.), McGraw-Hill, New York, 1975, pp. 115-155

[5] B. K. P. Horn and M. J. Brooks, eds., Shape from Shading, MIT Press, Cambridge, MA, 1989

[6] B. K. P. Horn and R. W. Sjoberg, Calculating the reflectance map, Applied Optics 18, 1770-1779 (1979)

[7] T. Jech, Set Theory, Academic Press, New York, 1978

[8] A. P. Pentland, Finding the illuminant direction, Journal of the Optical Society of America 72, 448-455 (1982)

[9] W. A. Veech, A Second Course in Complex Analysis, W. A. Benjamin, New York, 1967 